\title{
Gamificación en aplicaciones móviles para servicios bancarios de España
}

\section{Gamification in mobile applications for banking services in Spain}

\author{
Dr. Ángel Torres-Toukoumidis \\ Universidad de Huelva. España \\ angel.torres@alu.uhu.es \\ orcid.org/0000-0002-7727-3985
}

Dra. Pilar Marín-Mateos Universidad de Huelva. España marin@uhu.es orcid.org/0000-0003-3474-908x

\begin{abstract}
Resumen
Este estudio se basa en identificar la incorporación de elementos de juego en aplicaciones móviles del sector financiero español, específicamente se recoge una muestra de 38 aplicaciones pertenecientes a la banca móvil, que provienen de las tiendas AppStore del sistema operativo iOS y Google Play del sistema operativo Android excluyendo todas aquellas que no pertenecen a una entidad bancaria como es el caso de PayPal, FinTonic, Mapfre, Plus500, Trading 212 Forex \& Stocks, Carrefour Pass Móvil, y se le aplica la taxonomía de elementos lúdicos proveniente de Raftopoulos, Walz y Greuter (2015) organizado en propósito, audiencia, estrategia tecnológica, experiencia lúdica y mecánicas de juego. Los resultados demuestran que los propósitos de tales aplicaciones es fomentar la fidelidad del cliente, presentando soluciones operativas y sincronizaciones de carteras. Por su parte, la audiencia se orienta a un perfil de edad ubicado entre 26 y 35 años, mientras que la estrategia tecnológica responde primordialmente a la adopción de sistemas de seguridad y privacidad. En complemento, la experiencia lúdica va paulatinamente adoptándose por la comunicación móvil de las entidades bancarias, especialmente en cuanto a socialización, configuración personalizada del panel central. Por último, las mecánicas más utilizadas son la recolección de recompensas y aplicación de mecánicas de progresión.
\end{abstract}

\begin{abstract}
This study is based on identifying the incorporation of game elements in mobile applications of the Spanish financial sector, specifically a sample of 38 applications belonging to mobile banking, which come from the AppStore stores operating system iOS and Google Play operating system Android, excluding all those that do not belong to a banking entity such as PayPal, FinTonic, Mapfre, Plus500, Trading 212 Forex \& Stocks, Carrefour Mobile Pass, and the taxonomy of game elements from Raftopoulos, Walz and Greuter (2015) organized in purpose, audience, technological strategy, play experience and game mechanics. The results demonstrate that the purposes of such applications are to foster customer loyalty by presenting operational solutions and portfolio synchronizations. The audience is oriented to an age profile between 26 and 35 years, while the technology strategy responds primarily to the adoption of security and privacy systems. In addition, the ludic experience is gradually adopted by the mobile communication of banking entities, especially in socialization, personalized configuration of the central panel. Finally, the mechanics most used are the collection of rewards and application of mechanics of progression.
\end{abstract}

\section{Palabras clave | keywords}

Aplicación software, banco, comunicación móvil, España, instituciones financieras, juego. Software application, Bank, mobile communication, Spain, financial institutions, game. 


\section{Introducción}

El Instituto Nacional de Estadística (2015) establece que el 96,7\% de los españoles dispone de un teléfono móvil, mientras que el 22,7\% que utilizan internet han llevado a cabo actividades conectadas a la gestión de cuentas bancarias. Por su parte, Ditrendia (2015) presenta que alrededor de 15 millones de españoles han adoptado el uso de la banca móvil para manejar sus finanzas. La prospectiva sobre estos servicios estima que para el 2020 los teléfonos móviles inteligentes serán responsables del $80 \%$ del mercado bancario.

En diciembre de 2009, Caixabank fue la primera entidad financiera de España en sumarse a las plataformas de distribución digital como AppStore, Android Market, conocido actualmente como Google Play y App World de Blackberry, integrando entre sus funciones el chequeo de los balances, transferencias, gestión de cuentas, localización de oficinas y atención al cliente. Prácticamente cuatro años más tarde, en diciembre de 2013, aparece la primera aplicación móvil proveniente de un banco español que permitía la realización de pagos, cabe destacar que el servicio de pagos se emite a través de una aplicación distinta a la aplicación principal, es decir, el BBVA, pionero en este servicio, disponía una aplicación dedicada a la consulta y gestión bancaria y complementariamente presentó la aplicación BBVA Wallet admitiendo de eso modo la realización de pagos contactless a través de teléfonos móviles, abriendo así un nuevo campo acción para la conversión digital de la banca. Posteriormente, entidades como Caixabank, Bankia, Banco Sabadell, Banco Santander, se unen a dicha tendencia.

Según Rodrigues, Costa y Oliveira (2014) la banca electrónica, incluyendo la banca móvil ha estado experimentando con la inserción de elementos de juego, en otras palabras, la creación de una banca electrónica gamificada supone aumentar la fidelidad, tiempo y número de transacciones de los clientes.

\section{Banca móvil}

El comercio móvil, m-commerce, se presenta como la extensión del comercio electrónico que ha proporcionado mayor flexibilidad y movilidad en los servicios de localización, control de calidad e inversión (Varshney, 2008). Entre las razones que sustentan tal progreso está la amplia penetración del mercado de los celulares, junto con la estabilidad general de las tecnologías de comunicación móvil y las experiencias 
positivas producidas en su comercialización (Mallat, Rossi \& Tuunainen, 2004), efectivamente, el m-commerce ha hecho que las soluciones móviles sean aplicables a una variedad de servicios financieros experimentando cambios importantes en los últimos años. El sector bancario se ubica en los puestos de liderazgo en la adopción y utilización de tecnología móvil en los servicios financieros, generando un nuevo valor añadido a los clientes al reducir los costes y al mejorar la calidad de los canales de interacción (Pousttchi \& Schurig, 2004; Laukkanen, 2007).

Luo, Li, Zhang y Shim (2010) definen la banca móvil como un método innovador para acceder a servicios bancarios donde el cliente interactúa con un banco a través de un dispositivo móvil. Entre sus ventajas se encuentran la atemporalidad, la ubicuidad y las bajas tarifas, accediendo a múltiples bancos, cuentas y servicios financieros desde cualquier lugar, y a cualquier hora (Karjaluoto, 2002). En definitiva, el desplazamiento progresivo de los usuarios en el uso de los ordenadores hacia las aplicaciones de los dispositivos móviles, ha abierto una oportunidad que los bancos europeos han buscado rentabilizar (Gjino, \& Ilollari, 2014).

Entre los factores diferenciadores que se han integrado para potenciar la rentabilidad de la banca móvil se evidencia gamificación, big data, computación en la nube, robots móviles, análisis predictivos, softwares para el reconocimiento de voz, entre otros (Maldonado, 2013). En el primero de ellos, se constata la influencia positiva en la utilidad percibida y en las intenciones de los clientes (Rodrigues, Costa \& Oliveira, 2014).

\section{Gamificación}

En el campo de las nuevas tecnologías, el escenario europeo está presente esta vez mediante el programa de investigación e innovación Horizon 2020, en el que se propone el tema: Advanced digital gaming/ gamification technologies, para desarrollar nuevas metodologías, herramientas y evidencia científica en contextos digitales no lúdicos para el beneficio de gobiernos, empresas e individuos.

Todo fenómeno social que ejerce algún efecto en las personas, como es el caso de la gamificación, está orientado en base a principios que sustenten sus acciones. En este caso en particular se visualizan cinco clasificaciones enfocadas desde distintas perspectivas. Principios pregamificación, principios de gamificación, principios para los procesos de gamificación y principios para optimizar la gamificación. Así se dis- 
tinguen los de Coonradt y Nelson (2007), desarrollados antes de que se formalizara el término gamificación; los expresados por Ivanovna (2013) para adaptar la gamificación a la sociedad actual; los seis principios de la gamificación eficiente orientada a los usuarios, de Marache-Francisco y Brangier (2013); Oprescu, Jones y Katsikitis (2014) para la adopción de la gamificación en el trabajo.

Coonradt y Nelson (2007), antes que existiera el término gamificación, determina cinco principios que motivan la recreación dentro de un ambiente laboral:

1. Metas claramente definidas: Este principio afirma que las metas deben estar escritas, cada persona debe comprometerse a lograr sus propias metas para que se pueda obtener un beneficio colectivo, las metas deben ser positivas, medibles, realistas, obtenibles y específicas, las metas deben ser visibles como son los puntos, las metas deben tener una fecha de vencimiento, las metas deben producir cambios en la personalidad de las personas que participan y por último las metas deben tener beneficios complementarios.

2. Mejorar el registro de puntos y tarjetas de puntaje: El registro de puntos debe ser objetivo y simple, auto-administrado pero audita-do por sus pares, se busca que sea dinámico y que sirva de compa-ración personal con su rendimiento pasado.

3. Feedback frecuente: Refuerza comportamientos que deben ser repetidos, la cantidad apropiada de feedback la determina el que lo recibe, la habilidad de ofrecer feedback es una de las habilidades más importantes del ser humano, la persona que da el feedback siempre está en control, es mejor ofrecer un feedback negativo que no ofrecer feedback alguno.

4. Un mayor grado de elección personal. El sentido de autonomía es importante para todo ser humano, ya que refuerza sus actitudes y su poder de selección. Las metas pueden ser determinadas por otra persona, empresa, etc., pero el camino para lograrlo debe ser netamente personal.

5. Constante entrenamiento: Parte del entrenamiento debe ser un feedback continuo, evitar cambiar las reglas a mitad del juego, persistencia en las ideas, el sentimiento hacia una institución, marca, organización es aquella que produce la persona que realiza el entrenamiento. 
Coonradt y Nelson (2007) fundamenta sus estudios en los juegos deportivos para aplicar dinámicas de juego que motivaran y entretuvieran a las personas en las empresas.

Casi veinte años más tarde, Ivanovna (2013) defiende que las características inmanentes de un juego al apoyarse en el desarrollo de las nuevas tecnologías, son el sustento primario para formar los principios básicos de la gamificación: Participación voluntaria, libertad, vivir experiencias que producen emociones fuertes; Escape de la realidad a través de un mundo ilusorio, con reglas particulares y diversos escenarios; Los juegos se basan en procesos de autosuficiencia en lo que tienden a buscar la motivación intrínseca, sobre la motivación extrínseca.

Según Ivanovna (2013), estos serías los componentes actuales de las nuevas tecnologías en los cuales se apoyan los juegos:

- Interactividad: La interacción online ha producido un sistema de cooperación con objetos y sujetos dentro de las redes del espacio comunicativo. Los elementos de los juegos han servido como mecanismo de interacción debido a que estructura la actividad de las personas hacia un propósito donde resalta la simultaneidad.

- Fenómeno de la pantalla: El proceso de digitalización en la transmisión de información ha permitido que se emitan señales en tiempo real combinando sonidos, imágenes, textos, etc. Creando así un sentido de pertenencia.

- Virtualidad: El desarrollo de las nuevas tecnologías ha permitido el comportamiento voluntario hacia reglas obligatorias dentro de una existencia virtual, donde se adapta a mecánicas de juego para resolver problemas.

Ivanovna (2013), considera que el principio sobre el cual debe sustentarse la gamificación se obtiene a través de la combinación de las mecánicas de juego con el reconocimiento de los medios de comunicación. De acuerdo con su posición, estas pueden crear experiencias emocionales específicas en los jugadores. En consecuencia, si los medios de comunicación reconocen el valor de las mecánicas de juego y los incorporan, la interactividad puede alcanzarse en diferentes plataformas sin recurrir a cambios en la jugabilidad. En otras palabras, lo que ella plantea es que la transmedialidad es el principio fundamental de la gamificación.

Según el análisis realizado por Marache-Francisco y Brangier (2013), todo proceso de gamificación necesita precisar una serie de 
principios que guíen eficientemente la experiencia de los usuarios. Solo así se podrá materializar un conjunto de fases que satisfagan el proceso. Estos principios tienen una particularidad respecto a las demás, tienen una visión macro del proceso, no buscan articular los elementos de la gamificación como ocurre en las otras cuatro taxonomías, sino que contienen una base generalista, muy similar a la utilizada en el Know How empresarial.

- Libertad de elección: Darle al usuario la libertad de actuar voluntariamente, permitiéndole desactivar funciones y participar en la experiencia gamificada.

- Beneficios y significados: La influencia de la gamificación debe ser relevante tanto para los creadores como para los usuarios, a través de componentes que los motiven. En caso contrario, el uso del sistema no producirá influencia alguna, por ende, no se obtendrán beneficios.

- Experiencia personalizada: El sistema debe actuar sobre diferentes perfiles de usuarios para desencadenar actos que demuestren la aceptación general.

- Interacción a largo plazo: Las interacciones cambian. Aquí es importante tener en cuenta las teorías motivacionales.

- Efectos secundarios no deseados. Inducir al estrés, dar la sensación de falta de credibilidad y privacidad, la subjetividad con las recompensas, son factores que pueden jugar en contra de los sistemas gamificados.

- Asunto legal y ético: Existe un contexto legal sobre datos, privacidad e intereses de los usuarios que debe respetarse.

Estos principios, contribuyen a la posibilidad de emitir un proceso de gamificación que tenga como prioridad salvaguardar los intereses de todas las personas que participan. Sin importar el compromiso y la disposición inicial de los usuarios, el proceso debe ser igual para todos.

Desde otra perspectiva, Oprescu, Jones y Katsikitis (2014) adaptan la gamificación al trabajo. Sabemos a priori que el contexto hacia el cual dirigen sus principios, supone un alejamiento en un planteamiento general. No obstante, después de revisarlos, lo que ellos denominan trabajo, no implica necesariamente una empresa, una oficina, sino organizaciones, administraciones públicas. Entonces, de acuerdo con Oprescu, Jones y Katsikitis (2014) (2014) aumentar la productividad y los beneficios es proporcional al comportamiento de las personas hacia un fin en común. Ese fin no lo limitan, por tanto, puede involucrar necesidades sociales. 
En este caso son principios para entornos educacionales, colegios, universidades y espacios de enseñanza, que pueden ser de utilidad para motivar tanto a los estudiantes como a los profesores, mejorando en ambos su desempeño.

Atendiendo a las propuestas de Coonradt y Nelson (2007) al inicio de la gamificación; Ivanovna (2013) en el contexto de la gamificación; Marache-Francisco y Brangier (2013) en principios de gamificación efectiva; Oprescu, Jones y Katsikitis (2014) gamificación en el trabajo, junto con el Hype Cycle de Gartner (Rivera \& Van der Meulen, 2014) presentaba la gamificación como una tecnología emergente con una proyección de avance entre 2 a 5 años. Un año más tarde, la gamificación había desaparecido de este ciclo. Esta omisión se produce ya que la gamificación se traslada de tecnología emergente a una tecnología fija en sector productivo. Actualmente, la definición más reconocida en el entorno académico se refiere al uso de elementos de diseño de juego en contextos no lúdicos (Deterding, Dixon, Khaled \& Nacke, 2011).

En otras palabras, la gamificación se concibe como el proceso de utilizar elementos de juego en áreas no convencionales, entre ellas, el mercado financiero. De hecho, disciplinas como la administración de empresas y el marketing buscan apropiarse del término acercándolo a su terreno de estudio, Zichermann y Linder (2013), añaden una visión mercantilista considerándolo como la implementación de conceptos de diseño de juegos, programas de lealtad y economía conductual para impulsar la participación de los usuarios, mientras que Huotari y Hamari (2012) trasladan la conceptualización de la gamificación al marketing y publicidad, definiéndola como el proceso de mejorar un servicio para desarrollar experiencias lúdicas destinadas apoyar la creación de valor total del usuario.

El objetivo en el modo general se centra en el comportamiento de las personas, apelando a la persuasión para prolongar su participación dentro de un ámbito específico (Werbach \& Hunter, 2012). Sin embargo, en el caso de las empresas se trata de una estrategia para fidelizar clientes, mantener su interés sobre los productos y servicios, y premiar su compromiso con la marca, o bien como afirma Moiño (2013) mejorar la productividad de los empleados o influir en el comportamiento de los clientes, con el objetivo de generar beneficios reales para la empresa. Por otra parte, cuando se trata de buscar el bienestar social y cumplir con las necesidades de la sociedad, el objetivo de la gamificación cambia radi- 
calmente, enfocándose en aumentar el compromiso y motivación de las personas hacia una actividad haciéndola más divertida con la finalidad de estimular su aprendizaje (Landers \& Callan, 2011; Simões, Redondo \& Fernández-Vilas, 2012). En las nuevas tecnologías la dicotomía social permanece. Las empresas mantienen sus principios de acumulación de riqueza cuando los demás buscan una optimización del bienestar social.

Deterding, Dixon, Khaled y Nacke (2011) precisaron que, según los diseñadores de juego, la gamificación es añadir puntos, medallas y tabla de posiciones a las actividades mundanas de los usuarios. Dicha perspectiva ha encasillado la gamificación hacia las mecánicas relacionadas con los sistemas de recompensa, llegando a tal extremo que Zagalo y Oliveira (2014) la denominaron Pointsfication, haciendo referencia al uso de puntos como mecánica prioritaria. Si bien resulta cierto que los puntos, medallas y tabla de posiciones forman parte de las mecánicas de la gamificación, no son los únicos elementos que configuran su clasificación. De hecho, en la literatura actual pueden evidenciarse múltiples elementos de gamificación ordenados por diferentes taxonomías como Robinson y Bellotti, (2012); Thiebes, Lins y Basten, (2014): Werbach y Hunter, (2012); Zichermann y Cunningham, (2011). Esto supone una disyuntiva para la investigación ya que las clasificaciones contrastan entre sí, mezclando elementos de gamificación y de diseño de juego (Reeves \& Read, 2009), y a su vez aplicando aquellos que mejor se adaptaban a las propias temáticas de la investigación (Rai \& Beck, 2012; Dubbels, 2013). Pese a ello, la taxonomía presentada por Raftopoulos, Walz y Greuter (2015) abarca explícitamente la tecnología móvil y las empresas involucradas en el sector financiero.

\section{Material y métodos}

El objetivo de esta investigación consistió en identificar los elementos de gamificación en las aplicaciones móviles provenientes de entidades bancarias en España. Cada aplicación fue descargada y revisada desde el 10 de diciembre 2016 hasta el 10 de enero de 2017, siguiendo la taxonomía de gamificación propuesta por Raftopoulos, Walz y Greuter (2015), logrando visualizar el nivel de inserción de la gamificación en la banca móvil. 
La muestra se calculó tomando el universo de 500 aplicaciones móviles gratuitas más descargadas en la categoría finanzas. A partir de allí, se calculó el estimado del 95\% de nivel de confianza dentro de un intervalo \pm 1 resultando 38 aplicaciones provenientes de las tiendas AppStore del sistema operativo iOS y Google Play del sistema operativo Android excluyendo todas aquellas que no pertenecen a una entidad bancaria como es el caso de PayPal, FinTonic, Mapfre, Plus500, Trading 212 Forex \& Stocks, Carrefour Pass Móvil, Verse, entre otras.

\section{Tabla 1. Muestra seleccionada de aplicaciones móviles y} valoración en las stores

\begin{tabular}{|c|c|c|c|c|}
\hline $\mathrm{N}^{\circ}$ & Aplicación móvil & $\begin{array}{l}\text { Posición en } \\
\text { AppStore }\end{array}$ & $\begin{array}{c}\text { Posición en Google } \\
\text { Play }\end{array}$ & Fecha de lanzamiento \\
\hline & CaixaBank & 1 & 1 & 2009 \\
\hline & BBVA | Spain & 2 & 2 & 2010 \\
\hline & Santander & 3 & 6 & 2010 \\
\hline & CaixaBank Pay & 4 & 5 & 2016 \\
\hline & ING DIRECT Banca & 6 & 13 & 2014 \\
\hline & Banco Sabadell & 7 & 4 & 2010 \\
\hline & Bankia & 9 & 7 & 2010 \\
\hline & BBVA Wallet & 10 & 12 & 2013 \\
\hline & Twyp Cash & 11 & 8 & 2016 \\
\hline & Santander Wallet & 13 & 23 & 2015 \\
\hline & Línea Abierta Caixa & 15 & 19 & 2013 \\
\hline & Ruralvía & 16 & 17 & 2012 \\
\hline & WiZink & 17 & 39 & 2016 \\
\hline & $\begin{array}{l}\text { Banco Popular } \\
\text { Español }\end{array}$ & 18 & 14 & 2016 \\
\hline & ReciBox Caixa & 19 & 22 & 2013 \\
\hline & Twyp ING Group & 21 & 11 & 2015 \\
\hline & Sabadell Wallet & 22 & 46 & 2016 \\
\hline & ABANCA & 23 & 27 & 2009 \\
\hline & Cajamar & 24 & 33 & 2011 \\
\hline & imaginBank -Caixa & 25 & 44 & 2016 \\
\hline & Kutxabank & 26 & 16 & 2014 \\
\hline & UnicajaMóvil & 27 & 32 & 2012 \\
\hline & Ibercaja & 28 & 20 & 2012 \\
\hline
\end{tabular}




\begin{tabular}{|l|l|l|l|l|}
\hline$N^{\circ}$ & \multicolumn{1}{|c|}{ Aplicación móvil } & $\begin{array}{c}\text { Posición en } \\
\text { AppStore }\end{array}$ & $\begin{array}{c}\text { Posición en Google } \\
\text { Play }\end{array}$ & Fecha de lanzamiento \\
\hline & Bankia Wallet & 30 & 24 & 2014 \\
\hline & EVO Banco Móvil & 31 & 45 & 2012 \\
\hline & Bankintercard & 32 & 47 & 2016 \\
\hline & Bankintermovil & 33 & 35 & 2016 \\
\hline & KutxabankPay & 35 & 50 & 2016 \\
\hline & BBVA net cash & 36 & 63 & 2013 \\
\hline & BMN Banca Online & 37 & 38 & 2012 \\
\hline & Ruralvía wallet & 38 & 42 & 2016 \\
\hline & IKEA VISA-Caixa & 39 & 66 & 2015 \\
\hline & CajaSur & 41 & 29 & 2014 \\
\hline & EspañaDuero Móvil & 44 & 52 & 2013 \\
\hline & $\begin{array}{l}\text { OpenBank. } \\
\text { Santander }\end{array}$ & 46 & 43 & 2011 \\
\hline & Santander Empresas & 47 & 96 & 2015 \\
\hline & $\begin{array}{l}\text { Santander Money } \\
\text { Plan }\end{array}$ & 49 & 61 & 2016 \\
\hline & $\begin{array}{l}\text { Banca Online Liber- } \\
\text { bank }\end{array}$ & 50 & 40 & 2013 \\
\hline
\end{tabular}

Fuente: App Annie, 10 de diciembre de 2016

La taxonomía de Raftopoulos, Walz y Greuter (2015) presenta cinco elementos centrales:

- Propósito: fidelidad del cliente; venta y promoción; educación y reclutamiento; innovación y solución de problemas; bien común y desarrollo; y productividad y moral del personal.

- Audiencia: personal interno; clientes; proveedores; comunidad específica y público general.

- Estrategia tecnológica: juego digital; simulación digital; plataforma de venta; plataforma personalizada; modificación sencilla de las características del producto; modificaciones significativas en las características del producto; experiencias lúdicas con niveles básicos de tecnología; y experiencia lúdica con niveles altos de tecnología.

- Experiencia lúdica: adquisición de territorio; predicción; supervivencia; construcción; captura y evasión; intercambio; resolución de problemas; socialización; navegación espacial; destrucción, recolección; y carrera. 
- Mecánicas de juego: estatus, éxito, reconocimiento; puntos; experiencia; misiones; bienes reales y virtuales; medallas y trofeos; líderes de tabla, progresión y narrativa.

\section{Análisis y resultados}

Si bien el 100\% de las aplicaciones fomentan la fidelidad del cliente, dado que para acceder a las opciones de la interface el usuario necesita poseer una cuenta y estar registrado en el banco determinado. Un total de 29 aplicaciones (76\%) de la muestra seleccionada se orientan a la búsqueda de la innovación y solución de problemas facilitando la ubicación de sucursales bancarias y cajeros automáticos, realización de transferencias, recarga del teléfono móvil, atención al cliente, domiciliación y consulta inmediata de pagos y recibos. Esto significa que más de la mitad de las aplicaciones se orientan a la simplificación de las operaciones bancarias y al ahorro de tiempo de los clientes.

En consiguiente, venta y promoción se erige como otro de los propósitos más reiterados en las aplicaciones (24\%), esto se debe a la sincronización de las carteras electrónicas de las diferentes entidades bancarias con las tarjetas de crédito y débito del usuario, recibiendo ofertas exclusivas al realizar pagos a través de este canal, además de otros beneficios.

Indefectiblemente el 100\% que representa la audiencia de este tipo de aplicaciones son los clientes de cada banco y que a su vez estén interesados en llevar a cabo este tipo de gestiones mediante su teléfono móvil. Según Ditrendia (2015), el 91\% de españoles ubicados en el rango entre 26 y 35 años utilizan los celulares con frecuencia y mantienen el perfil de consumidor proclive a la descarga de este tipo de aplicaciones.

El 100\% de la muestra comparte el hecho de ser softwares para dispositivos móviles con alto nivel tecnología, donde se evidencian primordialmente la adopción de sistemas de seguridad y privacidad como encriptación de datos SSL, protección PIE\&Stacks, OTP, entre otros, y tecnología de geolocalización también conocida como LBS, para la ubicación de cajeros y sucursales y para activar funciones de la cartera móvil, las cuales también cuentan con NFC -Near Field Communication- que permite realizar pagos sin el contacto de los dispositivos. Por otro lado, son aplicaciones que necesitan una constante actualización, 21 de las 38 aplicaciones tuvieron su última actualización en diciembre 2016, mientras que Banco Santander, Kutxabank, Evo Banco Móvil y Santander Empresas, ya se han actualizado durante el transcurso del estudio en 
enero 2017. En complemento, también se observan simulaciones dentro de las aplicaciones revisadas, las aplicaciones de Santander Money Plan, Santander, Kutxabank, Ibercaja, CajaSur, Banco Popular y Cajamar permiten al cliente calcular bonificaciones, intereses, hipotecas, seguros, planes de pensiones, fondos de inversión, entre otros.

En total se visualizan tres elementos conducidos a la experiencia lúdica: socialización (100\%), construcción (36\%), y recolección (18\%).La socialización se presenta a través de tres modalidades, atención al cliente por parte de la entidad bancaria, lista de contactos permitiendo realizar transferencias entre sí, y redes sociales, compartiendo en información comercial de las entidades bancarias. En cambio, el término construcción se refiere en este caso a la configuración personalizada de los productos y servicios del cliente, modificando el orden, las notificaciones, la visualización de los mismos y estableciendo favoritos. Las entidades que disponen de esta experiencia son: Ibercaja, Santander, ING DIRECT Banca, BBVA, CaixaBank, Banco Popular, Banco Sabadell, Cajasur, Españaduero Móvil, Sabadell Wallet, Cajamar, BMN Banca Online, IKEA Visa-Caixa y Santander Money Plan. Respecto a la recolección como experiencia lúdica precisa la aplicación de sistemas de recompensa dentro de la aplicación. Las aplicaciones bancarias que explícitamente desarrollan este elemento son: Unicaja, WiZink, CajaSur, Españaduero Móvil, Banca Online Liberbank, Kutxabank, Bankintercard.

En esta sección, el 26\% de las aplicaciones móviles representadas por Unicaja, Ibercaja, Evo Banco Móvil, Wizink, Recibox Caixa, IKEA Visa, Ruralvía, Caixabank Pay, ImaginBank- Caixa y Santander Wallet, utilizan la mecánica de progresión como componente para la visualización de pago de créditos, saldo disponible y consumido, renta y previsión de gastos. Asimismo, el 15\% ofrece puntos intercambiables por descuentos, ofertas y regalos como se observa en los siguientes: Unicaja. BMN Banca Online, Españaduero Móvil, Banca Online Liberbank, CajaSur y Bankintercad. Por último, de manera innovadora la aplicación Evo Banco Móvil ha integrado tabla de posiciones entre inversionistas.

\section{Discusión y conclusiones}

Según Romero-Rodríguez, Torres-Toukoumidis y Aguaded (2017) la gamificación se identifica como catalizador del cambio social al otorgar un carácter interactivo y transversal a las experiencias. Por ello, a la luz de los datos obtenidos, se constata que el sector bancario español, incluyendo 
bancos tradicionales entre ellos Banco Santander, Banco Popular, BBVA, CaixaBank como bancos de reciente aparición en el mercado financiero como Liberbank, Evo Banco y KutxaBank acceden a la implementación de la gamificación en sus aplicaciones móviles. Específicamente, al encontrarse con el propósito objetivo de aumentar la fidelidad de los clientes y una audiencia segmentada entre 26 y 35 años, se concibe tres aristas de la experiencia lúdica, en primer lugar, socialización, que se refiere a la atención al cliente, transferencias entre las personas que forman parte de la lista de contactos del móvil e información comercial a través de las redes sociales, seguidamente se observa la arista denominada construcción que comprende la configuración personalizada del orden y visualización de los productos y servicios del cliente. Para culminar, la tercera arista de la experiencia lúdica actual de los bancos es la recolección, en la que se toma en cuenta la búsqueda y acceso basado en sistemas de recompensa. Si bien los resultados demuestran que su incorporación aún se mantiene a pequeña escala, la progresiva transformación digital de la banca española incluye a la gamificación dentro de las tendencias más efectivas en la próxima década (Gutiérrez-Rubí, 2015).

Esta investigación abre un espacio para diagnosticar patrones de uso de la gamificación en el sector bancario, midiendo su utilidad y efecto en la clientela. De igual modo, se recomienda a futuros investigadores el acoplamiento del factor estético entre los elementos, el cual fue omitido en la taxonomía de Raftopoulos, Walz y Greuter (2015) y juega un papel importante en la navegación por la interface de la banca móvil (Rodrigues, Costa \& Oliveira, 2014).

\section{Referencias}

Coonradt, C. A., \& Nelson, L. (2007). The Game of Work. Utah, EEUU: Gibbs Smith. Deterding, S., Dixon, D., Khaled, R., \& Nacke, L. (2011). From game design elements to gamefulness: defining gamification. International Academic MindTrek Conference: Envisioning Future Media Environments, 9-15. ACM.doi: 10.1145/2181037.2181040.

Ditrendia (2015). Informe ditrendia 2016: Mobile en España y en el mundo. Madrid, España: Tatum.

Dubbels, B. (2013). Gamification, Serious Games, Ludic Simulation, and other Contentious Categories. International Journal of Gaming and Computer-Mediated Simulations, 5(2), 1-19. doi: 10.4018/jgcms.2013040101.

Gjino, G., \& Ilollari, O. (2014). Mobile banking: near future of banking. Review of Applied Socio-Economic Research, 7(1), 43-51. Recuperado de https:/goo.gl/ xcrWbV. 
Gutiérrez-Rubí, A. (2015). La transformación digital y móvil de la comunicación política. Madrid, España: Fundación Telefónica.

Huotari, K., \& Hamari, J. (2012). Defining Gamification: A Service Marketing Perspective. Proceedings of the 16th International Academic MindTrek Conference, ACM, pp. 17-22. doi: http://dx.doi.org/10.1145/2393132.2393137.

Instituto Nacional de Estadística (2015). Encuesta sobre Equipamiento y Uso de Tecnologías de Información y Comunicación en los Hogares [Nota de prensa]. Recuperado de https://goo.gl/9kXIOa.

Ivanovna, I. (2013). Four Pillars of Gamification. Middle-East Journal of Scientific Research, 13, 149-152. doi: http://dx.doi.org/10.1002/9780470752210.ch12.

Karjaluoto, H. (2002). Electronic banking in Finland: Consumer beliefs, attitudes, intentions and behaviors. Finlandia: University of Jyväskylä.

Landers, R. N., \& Callan, R. C. (2011). Casual Social Games as Serious Games: The Psychology of Gamification in Undergraduate Education and Employee Training. Serious games and edutainment applications, 399-423. doi: http://dx.doi. org/10.1007/978-1-4471-2161-9_20.

Laukkanen, T. (2007). Internet vs mobile banking: comparing customer value perceptions. Business Process Management Journal, 13(6), 788-797. doi: 10.1108/14637150710834550.

Luo, X., Li, H., Zhang, J., \& Shim, J. P. (2010). Examining multi-dimensional trust and multi-faceted risk in initial acceptance of emerging technologies: An empirical study of mobile banking services. Decision support systems, 49(2), 222-234. doi: 10.1016/j.dss.2010.02.008.

Mallat, N., Rossi, M., \& Tuunainen, V. K. (2004). Mobile banking services. Communications of the ACM, 47(5), 42-46. doi: 10.1145/986213.986236.

Marache-Francisco, C., \& Brangier, E. (2013). Perception of Gamification: Between Graphical Design and Persuasive Design. Design, User Experience, and Usability. Health, Learning, Playing, Cultural, and Cross-Cultural User Experience, 558-567. Springer Berlin Heidelberg. doi: http://dx.doi.org/10.1007/978-3642-39241-2_61.

Moiño, J. P. (2013). 'Gamification': aprenda a jugar sus cartas digitales. Harvard Deusto Marketing y Ventas, (115), 16-21. (http://goo.gl/f4zW5b) (2016-03-07).

Maldonado, L. (2013). La Banca, en la encrucijada. El futuro del sector financiero español en el mundo global. Madrid: PWC.

Oprescu, F., Jones, C., \& Katsikitis, M. (2014). I play at work. Ten principles for transforming work processes through gamification. Frontiers in Psychology, 14 (5) doi: http://dx.doi.org/10.3389/fpsyg.2014.00014.

Pousttchi, K., \& Schurig, M. (2004). Assessment of today's mobile banking applications from the view of customer requirements. In System Sciences, 2004. Proceedings of the 37th Annual Hawaii International Conference (pp. 10-15). IEEE. doi: 10.1109/hicss.2004.1265440

Rai, D., \& Beck, J. E. (2012). Math learning environment with game-like elements: an incremental approach for enhancing student engagement and learning effectiveness. In International Conference on Intelligent Tutoring Systems (pp. 90-100). 
Springer Berlin Heidelberg. doi: 10.1007/978-3-642-30950-2_13.

Reeves, B. \& Read, L. (2009). Total Engagement. Cambridge, EEUU: Harvard Business Press.

Rivera, J., \& Van der Meulen, R. (2014). Gartner's 2014 hype cycle for emerging technologies maps the journey to digital business. Connecticut, EEUU: Gartner Group.

Raftopoulos, M., Walz, S., \& Greuter, S. (2015). How enterprises play: Towards a taxonomy for enterprise gamification. Conference: Diversity of Play: Games-Cultures-Identities. DiGRA. Recuperado de https://goo.gl/3PD4f9.

Robinson, D., \& Bellotti, V. (2013). A preliminary taxonomy of gamification elements for varying anticipated commitment. Proceedings CHI 2013 Workshop on Designing Gamification: Creating Gameful and Playful Experiences. ACM. Recuperado de https://goo.gl/hElEnm.

Rodrigues, L. F., Costa, C. J., \& Oliveira, A. (2014). How gamification can influence the web design and the customer to use the e-banking systems. In Proceedings of the International Conference on Information Systems and Design of Communication (pp. 35-44). ACM. doi: 10.1145/2618168.2618174.

Romero-Rodríguez, L. M., Torres-Toukoumidis, Á., \& Aguaded, I. (2017). Ludificación y educación para la ciudadanía. Revisión de las experiencias significativas. Educar, 53(1), 109-128. doi: 10.5565/rev/educar.846.

Simões, J., Redondo, R. D., \& Vilas, A. F. (2013). A social gamification framework for a K-6 learning platform. Computers in Human Behavior, 29(2), 345-353. doi: http://dx.doi.org/10.1016/j.chb.2012.06.007.

Thiebes, S., Lins, S. \& Basten, D. (2014). Gamifying Information Systems- A Synthesis of Gamification Mechanics and Dynamics. Twenty Second European Conference on Information Systems. ECIS. doi: https://goo.gl/uyOYqK.

Varshney, U. (2008). A middleware framework for managing transactions in grouporiented mobile commerce services. Decision Support Systems, 46(1), 356-365. doi: $10.1016 /$ j.dss.2008.07.005.

Werbach, K., \& Hunter, D. (2012). For the Win: How Game Thinking Can Revolutionize your Business. Pennsylvania, EEUU: Wharton Digital Press.

Zagalo, N. y Oliveira, S. (2014). Perspectivas sobre a Gamificação: um fenómeno que quer gerar envolvimento. Braga, Portugal: Centro de Estudos de Comunicação e Sociedade, Universidade do Minho. doi: https://goo.gl/gHIzAS.

Zichermann, G., \& Cunningham, C. (2011). Gamification by Design: Implementing Game Mechanics in Web and Mobile Apps. Massachusetts, EEUU: O'Reilly Media, Inc.

Zichermann, G., \& Linder, J. (2013). The Gamification Revolution: How Leaders Leverage Game Mechanics to Crush the Competition. Nueva York, EEUU: McGrawHill Professional. 